\title{
Single Oral Administration of Anthocyanin Rescues Smoking-Induced Endothelial Dysfunction in Young Smokers but Facilitates Oxidative Stress in Non-Smokers
}

\author{
Akane Yoshizaki', Toshiko Tomisawa², Tomohiro Osanai², Naoki Nanashima3 ${ }^{3}$, Maiko Kitajima², \\ Kasumi Mikami' ${ }^{2}$, Toshifumi Fujita ${ }^{4}$, Hayato Maeda ${ }^{5}$, Yoji Kato ${ }^{6}$ \\ ${ }^{1}$ Hirosaki University Medical Hospital, Hirosaki, Japan \\ ${ }^{2}$ Department of Nursing Science, Hirosaki University Graduate School of Health Sciences, Hirosaki, Japan \\ ${ }^{3}$ Department of Biomedical Sciences, Hirosaki University Graduate School of Health Sciences, Hirosaki, Japan \\ ${ }^{4}$ Department of Disability and Health, Hirosaki University Graduate School of Health Sciences, Hirosaki, Japan \\ ${ }^{5}$ Faculty of Agriculture and Life Science, Hirosaki University, Hirosaki, Japan \\ ${ }^{6}$ Faculty of Education, Hirosaki University, Hirosaki, Japan \\ Email: akaney.516@gmail.com, tmtott@hirosaki-u.ac.jp, osanait@hirosaki-u.ac.jp,nnaoki@hirosaki-u.ac.jp, \\ kitajima@hirosaki-u.ac.jp, k-mikami@hirosaki-u.ac.jp, pttoshi@hirosaki-u.ac.jp, hayatosp@hirosaki-u.ac.jp, \\ ykato@hirosaki-u.ac.jp
}

How to cite this paper: Yoshizaki, A., Tomisawa, T., Osanai, T., Nanashima, N., Kitajima, M., Mikami, K., Fujita, T., Maeda, H. and Kato, Y. (2018) Single Oral Administration of Anthocyanin Rescues Smoking-Induced Endothelial Dysfunction in Young Smokers but Facilitates Oxidative Stress in Non-Smokers. Food and Nutrition Sciences, 9, 179-190.

https://doi.org/10.4236/fns.2018.93014

Received: February 2, 2018

Accepted: March 5, 2018

Published: March 8, 2018

Copyright $\odot 2018$ by authors and Scientific Research Publishing Inc. This work is licensed under the Creative Commons Attribution International License (CC BY 4.0).

http://creativecommons.org/licenses/by/4.0/

(c) (i) Open Access

\begin{abstract}
Objectives: Smoking causes endothelial dysfunction, but it still remains unclear whether oral administration of anthocyanin brings benefits to endothelial function and redox states in young healthy smokers. We tested the hypothesis that supplement anthocyanin may rescue endothelial dysfunction and redox states in young healthy smokers. Methods: Young healthy male non-smoker (n $=8)$ and smokers $(n=14)$ were enrolled to measure the derivatives of reactive oxygen metabolites (d-ROMs) and biological antioxidant potential (BAP) levels and brachial artery flow-mediated dilation (FMD) before and 2 hours after one cigarette smoking or resting under administration of blackcurrant supplements (anthocyanins $210 \mathrm{mg}$ ) or not. Results: FMD, d-ROMs, and BAP at baseline were similar between non-smokers and smokers. One cigarette smoking induced a decrease in FMD at 2 hours $(9.0 \% \pm 2.6 \%$ vs $7.5 \% \pm 2.3 \%$, $\mathrm{p}<0.05)$ without affecting plasma levels of d-ROMs and BAP in young healthy smokers. Single oral administration of anthocyanin at $210 \mathrm{mg}$ abolished smoking-induced decrease in FMD $(8.3 \% \pm 3.4 \%$ vs $9.5 \% \pm 3.6 \%, \mathrm{p}=$ ns) without changes in plasma levels of d-ROMs and BAP in young smokers. In non-smokers, however, administration of anthocyanin at $210 \mathrm{mg}$ slightly but significantly elevated plasma level of d-ROMs at 2 hours $(249.6 \pm 30.3$ vs
\end{abstract}


$265.5 \pm 36.3 \mathrm{U}$ CARR, $\mathrm{p}<0.05)$ without affecting plasma level of BAP and FMD. Conclusions: Single oral administration of anthocyanin rescued smoking-induced endothelial dysfunction in young healthy smokers, but facilitated oxidative stress in non-smokers. When anthocyanin is taken as supplements, it seems important to pay attention to the dose and timing of administration.

\section{Keywords}

Blackcurrant, Anthocyanin, Young Health Smoker, Oxidative Stress, Antioxidants

\section{Introduction}

Lifestyle-related disease has become an object of public concern, and arteriosclerosis is attributable to the pathogenesis of lifestyle-related diseases such as cardiovascular disease. Smoking is predisposed to arteriosclerosis and regarded as one of the major risk factors for the development of cardiovascular diseases [1]. Smoking comprises not only free radicals such as superoxide and reactive oxygen but also several other materials of carbon monoxide, nitrogen, and nicotine [2]. Reactive oxygen species and reactive nitrogen species constituents in both the tar and gas phases of smoke are associated with various diseases like arteriosclerosis, diabetes or cancer [3]. The World Health Organization predicted that deaths related to cigarette smoke will increase up to eight million individuals per year worldwide [4]. Furthermore, recent evidence showed that young smokers have a higher risk to develop vascular functional and histological abnormalities which are early hallmarks of atherosclerosis [5]. Therefore, it is important to develop new therapeutic strategies to prevent negative effects on the cardiovascular system by smoking.

Blackcurrant is rich in many health-beneficial substances, such as polysaccharides, flavonoids and anthocyanins [6]. Of these, anthocyanins are known to undergo gut microbial conversion into corresponding aglycones or anthocyanidins [7] [8]. Anthocyanin-rich bilberry and blackcurrant diets alleviate behavioral abnormalities in a mouse model of Alzheimer's disease [9]. Further, blackcurrant anthocyanins provide a number of health benefits, including improved blood flow and decreased inflammatory marker levels [10]. These findings suggest that blackcurrants may prohibit severe arteriosclerosis when taken as antioxidant supplements. Indeed, habitual antioxidant dietary intake was shown to provide potential benefits for secondary prevention of cardiovascular diseases [11]. However, the beneficial effects of antioxidant with respect to cancer were achieved by the diet itself and not by supplements [12]. Meta-analyses of randomized controlled trials also showed no relationship between the consumption of antioxidant supplements and cancer risk [13]. Thus, it still remains unclear whether supplements of anthocyanin are beneficial in healthy young population. In the present study, we examined the effect of intake of blackcur- 
rant anthocyanin supplements on endothelial function and oxidative stress markers after cigarette smoking in healthy young adults.

\section{Material and Methods}

\subsection{Materials and Reagents}

The blackcurrant supplement, which was made with solvent free, freeze-dried extraction methods to preserve quality, was purchased from Just the Berries Limited, New Zealand. It consists of not lower than $35.0 \mathrm{~g}$ total anthocyanin, 13.6 $\mathrm{mg}$ carbohydrate, $0.5 \mathrm{~g}$ lipid, and $0.1 \mathrm{~g}$ protein in total $100 \mathrm{~g}$, and energy is 1650 $\mathrm{Kj}(394 \mathrm{Kcal}) / 100 \mathrm{~g}$.

\subsection{Subjects}

Young healthy male non-smoker ( $\mathrm{n}=8$, mean age $23 \pm 2$ years) and smokers ( $\mathrm{n}$ $=14$, mean age $22 \pm 1$ years) were recruited from students of Hirosaki University. Exclusion criteria are having any disease such as hypertension, hyperlipidemia or diabetes, and a weight reducing dietary regimen or taking any dietary supplements. All subjects were asked to complete a health and lifestyle questionnaire, and suitable subjects were asked to sign an informed consent form. All study protocols were approved by The Committee of Medical Ethics of Hirosaki University Graduate School of Medicine, Hirosaki, Japan (2013-357).

\subsection{Study Design}

The subjects were asked to follow a low-flavonoid diet for 3 days before each determination and to refrain from taking hard exercise (Figure 1). They were also asked to getting adequate sleep before the determination. At least for 3 hours prior to testing, neither eating anything nor drinking coffee or tea was permitted in all subjects, and antioxidant vitamins were prohibited for the whole day before testing. Smokers were refrained from smoking at least for 3 hours before arrival in the laboratory. The subjects were examined at the spine position after 15 -minutes rest in quiet and air-conditioned room $\left(22^{\circ} \mathrm{C}-26^{\circ} \mathrm{C}\right)$.

Brachial artery flow-mediated dilation (FMD) was measured, and blood samples were taken from cubital vein to measure free radical and antioxidant capacity. Smokers were undergoing two series of experiments: one cigarette smoke alone, and take blackcurrant supplements after one cigarette smoke. Non-smokers were undergoing two series of experiments: no supplements or blackcurrant supplements alone.

Brachial artery FMD were measured two hours after one cigarette smoking and/or blackcurrant supplements alone. Blood samples were taken two hours after one cigarette smoking and/or blackcurrant supplements alone.

\subsection{Derivatives of Reactive Oxygen Metabolites and Biological Antioxidant Potential Levels}

The derivatives of reactive oxygen metabolites (d-ROMs) and biological antioxidant 


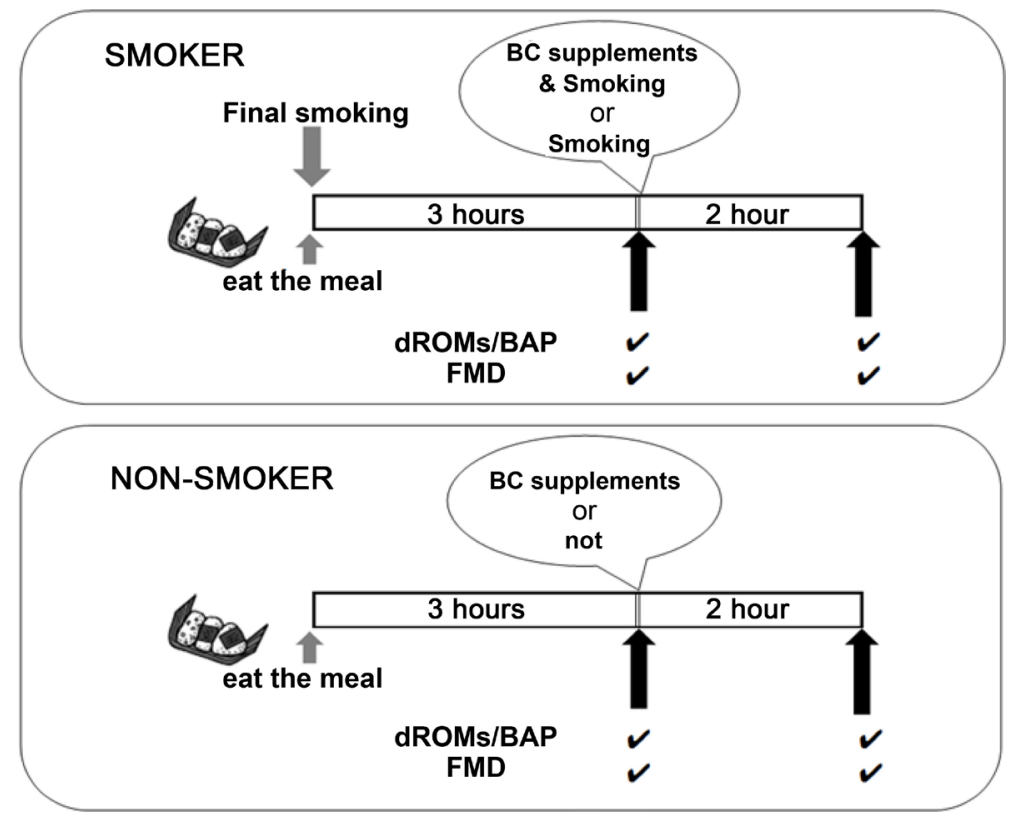

Figure 1. Protocol for this study.

potential (BAP) levels were measured using a Free Radical Elective Evaluator (Wismerll Co. Ltd, Tokyo, Japan) [14]. The d-ROMs measurements were determined based on the ability of transition metals to catalyze the formation of colored free radicals (detection at $505 \mathrm{~nm}$ ). The results were expressed in Cartelli units (U.CARR), where $1 \mathrm{U} . \mathrm{CARR}=0.8 \mathrm{mg} / \mathrm{L}$ of $\mathrm{H}_{2} \mathrm{O}_{2}$. The normal reference level of d-ROMs was 250 to 300 U.CARR [14]. To obtain the BAP measurements, the blood samples were added to a solution containing $\mathrm{FeC1}_{3}$ bound to a chromogenic substrate (AT, a derivative of thiocyanate). $\mathrm{Fe}^{3+}$ reduction to $\mathrm{Fe}^{2+}$ caused a chromatic change that was directly proportional to the plasma serum reactive oxygen species reduction, which was measured at $505 \mathrm{~nm}$ using a photometer. Blood aliquots at $10 \mu \mathrm{l}$ were mixed with the $\mathrm{FeCl}_{3}$ solution and incubated for $5 \mathrm{~min}$ at $37^{\circ} \mathrm{C}$ before the photometric analysis. BAP/d-ROM ratio represents the level of potential oxidative stress.

\subsection{Measurement of FMD}

Endothelium-dependent vasodilation and endothelium-independent vasodilation were measured according to methods that have been described previously. Brachial artery FMD was measured by a trained technician according to the guidelines for the ultrasound assessment. A linear-array transducer operating at $10 \mathrm{MHz}$ was used to acquire longitudinal images of the right brachial artery. A standard blood pressure cuff was positioned around the right arm, $5 \mathrm{~cm}$ below the antecubital fossa, and the artery was imaged 5 to $9 \mathrm{~cm}$ above the antecubital fossa. After baseline images were obtained, the cuff was inflated to $30 \mathrm{mmHg}$ above systolic blood pressure for 5 minutes. The diastolic pre-beat diameter of the brachial artery was determined semi-automatically using an instrument equipped with software for monitoring the brachial artery diameter (Unex Co. 
Ltd., Nagoya, Japan), and the validity and reproducibility of the equipment were confirmed. The participants rested on beds in supine position for at least $20 \mathrm{~min}$ before the study.

\subsection{Statistical Analysis}

Results were expressed as mean \pm SEM. Data were analyzed by using IBM SPSS Statistics 22.0. Statistical significance was defined as $\mathrm{p}<0.05$. For the analysis of between groups with or without taking blackcurrant supplements, a two-way repeated-measures ANOVA was applied. The intra-group comparison between pre-intervention and post-intervention was done by the Bonferroni correction for multiple comparisons.

\section{Results}

\subsection{Oxidative Stress, Antioxidant Capacity, and FMD after One Cigarette Smoking in Young Smokers and Resting in Non-Smokers}

As shown in Figures 2(a)-(d), d-ROMs, BAP, BAP/d-ROMs ratio, and FMD at baseline were all similar between young healthy non-smokers and smokers.

Figure 3 shows changes in d-ROMs (a), BAP (b), ratio of BAP/d-ROMs (c), and FMD (d) after one cigarette smoking in smokers and resting in non-smokers under no blackcurrant supplements. None of d-ROMs, BAP, and the ratio of

(a)

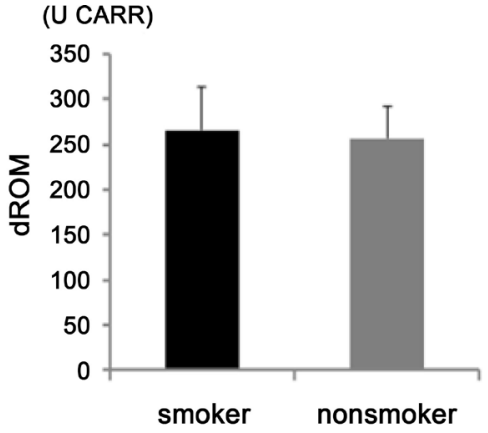

(c)

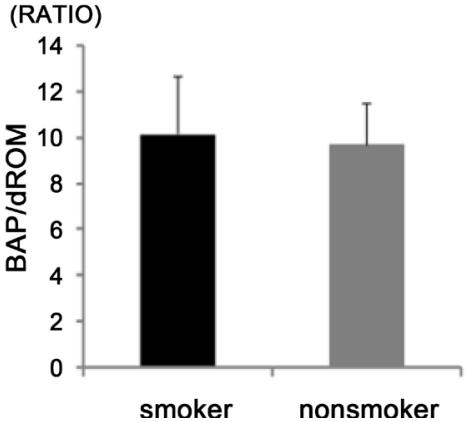

(b)

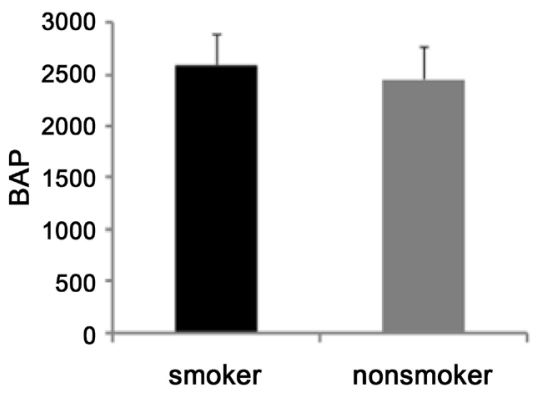

(d)

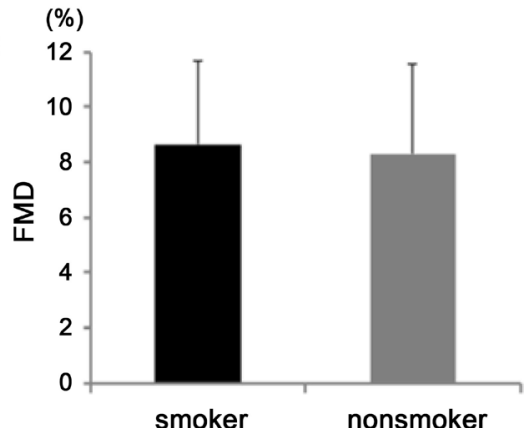

Figure 2. Baseline derivatives of reactive oxygen metabolites (d-ROMs) (a), biological antioxidant potential (BAP) (b), and ratio of $\mathrm{BAP} / \mathrm{d}-\mathrm{ROMs}$ (c), brachial artery flow-mediated dilation (FMD) (d) in young healthy smokers and non-smokers. Values are indicated as the means \pm standard errors. There are no significant difference between smokers and non-smokers by Student t-test. 


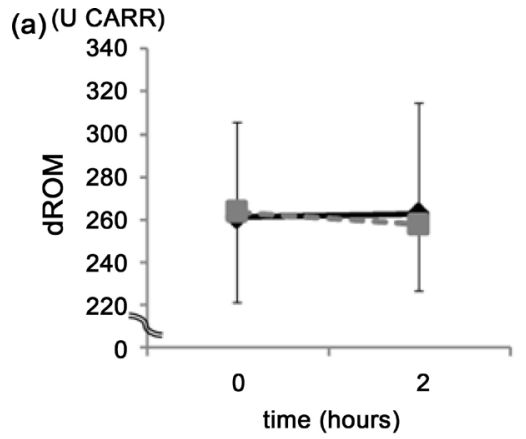

(c) (RATIO)

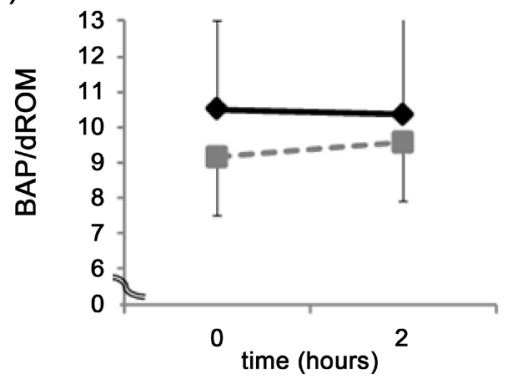

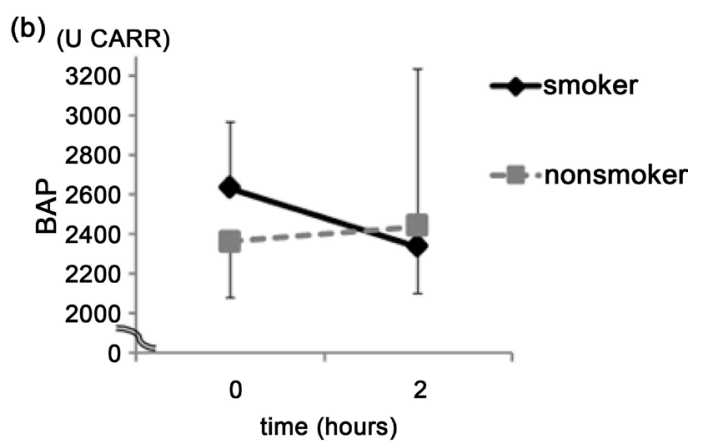

(d)

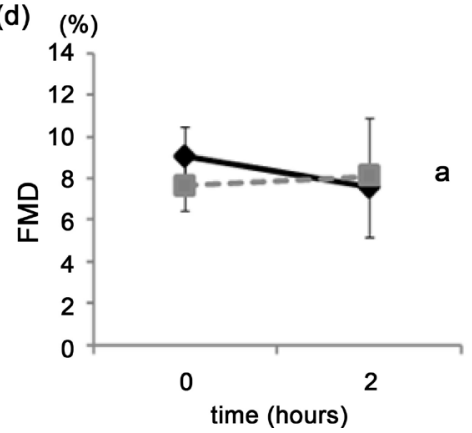

Figure 3. Changes in derivatives of reactive oxygen metabolites (d-ROMs) (a), biological antioxidant potential (BAP) (b), ratio of BAP/d-ROMs (c) and brachial artery flow-mediated dilation (FMD) (d) after one cigarette smoking in smokers and resting in non-smokers without blackcurrant supplements. Dotted line: nonsmoker. Solid line: smoker. Values are indicated as the means \pm standard errors. Difference in changes from baseline to 2 hours between groups was assessed by two-way repeated measure ANOVA followed by Bonferroni post hoc tests. Differences in changes in FMD from baseline to 2 hours are indicated by a for $\mathrm{p}<0.05$ by two way repeated measure ANOVA.

BAP/d-ROMs was changed 2 hours after one cigarette smoking in young healthy smokers (d-ROMs: $260.8 \pm 45.0$ vs $262.3 \pm 52.3$ U CARR in Figure 3(a), BAP: $2631.8 \pm 332.8$ vs $2336.4 \pm 898.0$ U CARR in Figure 3(b), and BAP/d-ROMs: $10.5 \pm 2.5$ vs $10.3 \pm 3.6$ in Figure 3(c), black solid lines). As shown in gray dotted lines (Figure 3(a) and Figure 3(b)), neither d-ROM nor BAP was changed after 2-hour resting in young healthy non-smokers. FMD was decreased from $9.0 \pm$ $2.6 \%$ to $7.6 \% \pm 2.4 \% 2$ hours after one cigarette smoking in young healthy smokers $(\mathrm{p}<0.05$, black solid line in Figure $3(\mathrm{~d})$ ). In non-smokers (gray dotted line in Figure 3(d)), FMD was unchanged during 2-hour resting.

\subsection{Effect of Blackcurrant Supplements on Oxidative Stress, Antioxidant Capacity, and FMD in Young Smokers and Non-Smokers}

Figure 4 shows changes in d-ROMs (a), BAP (b), ratio of BAP/d-ROMs (c) and FMD (d) 2 hours after one cigarette smoking in smokers and resting in non-smokers under blackcurrant supplements. The plasma value of d-ROMs was increased from $249.6 \pm 30.3$ to $260.5 \pm 49.3$ U CARR 2 hours after taking blackcurrant supplements and resting in young healthy non-smokers $(\mathrm{p}<0.05$, gray dotted lines in Figure 4(a)). Antioxidant capacity BAP was unchanged by 

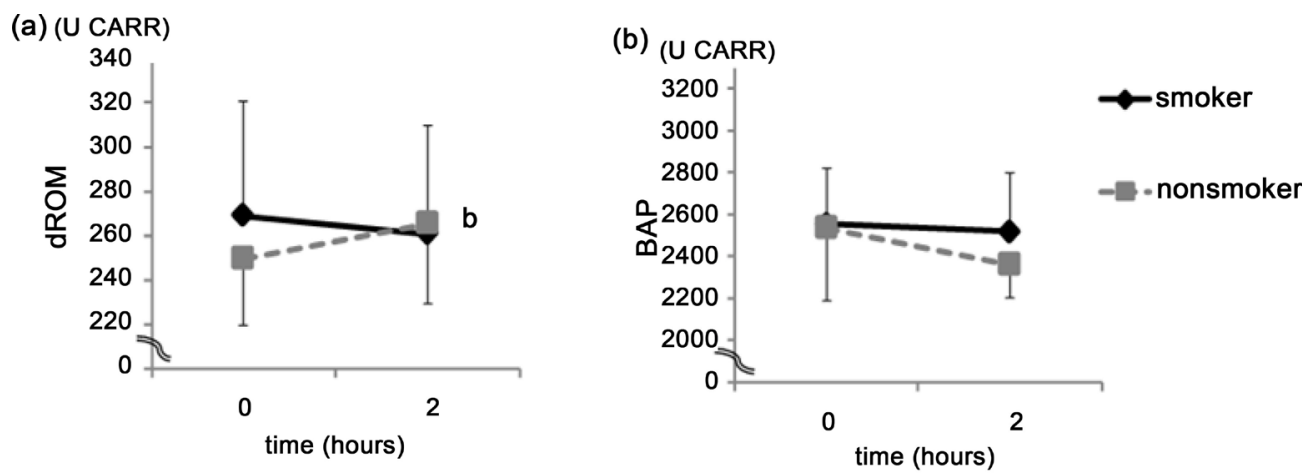

(c) (RATIO)
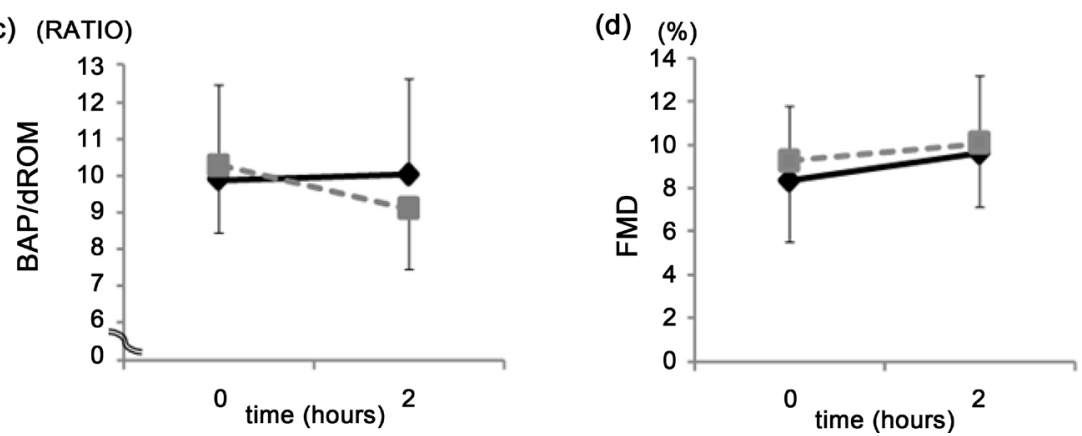

Figure 4. Changes in derivatives of reactive oxygen metabolites (d-ROMs) (a), biological antioxidant potential (BAP) (b), ratio of BAP/d-ROMs (c) and brachial artery flow-mediated dilation (FMD) (d) after one cigarette smoking in smokers and resting in non-smokers with blackcurrant supplements. Dotted line: nonsmoker. Solid line: smoker. Values are indicated as the means \pm standard errors. Difference in changes from baseline to 2 hours between groups was assessed by two-way repeated measure ANOVA followed by post hoc tests. Differences in changes in d-ROMs from baseline to 2 hours are indicated by $b$ for $p<0.05$ by two way repeated measure ANOVA.

taking blackcurrant supplements (gray dotted lines in Figure 4(b)). In young healthy smokers, neither d-ROMs nor BAP was changed 2 hours after one cigarette smoking under blackcurrant supplements (black solid lines in Figure 4(a) and Figure 4(b)). Single oral administration of anthocyanin at $210 \mathrm{mg}$ abolished one cigarette smoking-induced decrease in FMD $(8.3 \% \pm 3.4 \%$ vs $9.6 \% \pm 3.6 \%$, p $=\mathrm{ns}$ ) in smokers (black solid line in Figure $4(\mathrm{~d})$ ). FMD was unchanged after 2-hour resting in non-smokers under blackcurrant supplements (gray dotted line in Figure $4(d))$.

\section{Discussion}

The major findings of this study are as follows. Oxidative stress d-ROMs, antioxidant capacity BAP, and endothelial function at baseline were similar between young healthy smokers and non-smokers. One cigarette smoking induced transient endothelial dysfunction without affecting oxidative stress and antioxidant capacity in young healthy smokers. Single oral administration of anthocyanin rescued smoking-induced endothelial dysfunction without affecting oxidative stress and antioxidant capacity in young healthy smokers, but facilitated oxidative stress in young healthy non-smokers.

Oxidative stress d-ROMs, antioxidant capacity BAP, and endothelial function 
assessed by FMD measurement were all similar between young healthy smokers and non-smokers. In clinical studies, long-term exposure to cigarette smoke was reported to induce systemic lipid peroxidation with depletion of antioxidants such as vitamins $\mathrm{A}$ and $\mathrm{C}$ and elevation of inflammatory responses including C-reactive protein, fibrinogen, and interleukin (IL)-6 [15]. However, in short-term smokers, higher glutathione (GSH) levels as antioxidant capacity were observed in erythrocytes compared with non-smokers [16]. In response to cigarette smoking, $\gamma$-glutamyl cysteine ligase relating to GSH synthesis is upregulated in erythrocytes and results in increased production of GSH [17]. Thus, it is of significance that the level of antioxidant capacity BAP in young healthy smokers is located at the level between short-term and long-term smokers. This also suggests that an adaptive response to the continuous exposure to oxidants in cigarette smoking may be activated acutely promptly but declined chronically easily. In young healthy smokers, the maintained antioxidant defense system may lead to similar levels of baseline oxidative stress and endothelial function compared with those in non-smokers. In regard with other adaptive response to cigarette smoking, the lower IL-6 concentration in broncho-alveolar lavage fluid and the higher salivary superoxide dismutase activity were reported in healthy smokers [18] [19].

\subsection{Effect of One Cigarette Smoking on Redox State and Endothelial Function in Young Healthy Smokers}

Cigarette smoke oxidants can directly impair endothelial function, primarily through a lack of nitric oxide bioavailability which results both from decreased nitric oxide formation by the dysfunctional endothelium and increased nitric oxide scavenging by oxygen free radicals [20]. We previously confirmed that peroxidized LDLs with four kinds of hydroperoxidized cholesteryl linoleate were present in the plasma of young heavy smokers with more than 30 cigarettes/day over 5 years [21]. Thus the d-ROMs method to measure lipid peroxidation seems to be suitable system used. In the present study, we showed that one cigarette smoking did not affect the serum levels of d-ROMs and BAP in young healthy smokers. Lykkesfeldt et al. reported that smoking induces lipid peroxidation and increases the plasma levels of malondialdehyde in comparison to non-smokers [22]. The increase in plasma lipid peroxidation is observed in patients with stable ischemic heart disease [23], and is involved in augmented myocardial ischemia followed by reperfusion therapy [24]. In the young healthy smokers who may be still protected by an adaptive response in antioxidant system, however, single cigarette smoking might be unable to increase the level of plasma oxidative stress. In this study, endothelial function was decreased after one cigarette smoking in young healthy smokers. In general, endothelial dysfunction is one of the earliest pathological events that occur in the development of cardiovascular diseases [25], and decreased FMD is involved in endothelial dysfunction. Thus, it is likely that transient endothelial dysfunction induced by 
one cigarette smoking is caused by a lack of nitric oxide bioavailability presumably which results from decreased nitric oxide formation by dysfunctional endothelium rather than the increased nitric oxide scavenging by oxygen free radicals.

\subsection{Anthocyanin Supplements Increase Oxidative Stress in Young Healthy Non-Smokers}

In general, the beneficial effects of antioxidant-rich food with respect to cancer are widely reported [26] [27]; but these benefits are achieved by the diet itself and not by supplements [12]. Moreover, the dietary intake involves low doses of various antioxidants and not high doses of a single antioxidant. Indeed, two antioxidant-rich diets were found to be safe in a randomized controlled trial in male smokers [28].

The potential efficacy of dietary antioxidants at protecting against smoking-induced oxidative stress was indicated as follows. Decrease in plasma vitamin $\mathrm{C}$ was ameliorated by supplements of a vitamin cocktail containing vitamin C, $\boldsymbol{\alpha}$-tocopherol, and folate in smokers [29]. Vitamin C supplements were effective at reducing levels of lipid peroxidation in smokers [30]. Antioxidant supplements altered plasma lipid profiles in smokers in a manner consistent with the reduction in oxidative stress [31]. However, disease outcome of antioxidant clinical trials have provided less than anticipated results [32], and meta-analyses of randomized controlled trials for high doses of antioxidants except polyphenols found no overall relationship between the consumption of antioxidant supplements and cancer risk [13].

To date, in young healthy smokers who are at lower risk than old continuous smokers but maintain a higher risk than non-smokers, the clinical efficacy of dietary or supplementary antioxidants remains to be established. It is noted that single oral administration of anthocyanin unexpectedly elevated the serum level of d-ROMs slightly in young healthy non-smokers. In contrast, there was no effect of anthocyanin supplements on its level in young healthy smokers who were already protected by an adaptive response in antioxidant system. Furthermore, endothelial function was unchanged during two hours with or without blackcurrant supplements in non-smokers. Taken together, these suggest that an excessive increase in antioxidant system by single oral administration of anthocyanin may facilitate oxidative stress as a reactive response, thereby the state of redox balance is unchanged compared with that before administration of anthocyanin. To our knowledge, this is the first report that single oral administration of anti-oxidant supplements could cause the unexpected increase in the serum radical level in young healthy non-smokers.

\section{Acknowledgements}

The authors thank Mr. Syuhei Yamada (Hirosaki University) and Mr. Hiroshi Tomisawa (Central Chemical Co., Ltd.) for their support. This work was sup- 
ported in part by Grant-in-Aid for Scientific Research (C) (16K00844) and a Hirosaki University Grant for Exploratory Research by Young Scientists and Newly-appointed Scientists.

\section{References}

[1] Teo, K.K., Ounpuu, S., Hawken, S., Pandey, M.R., Valentin, V., Hunt, D., Diaz, R., Rashed, W., Freeman, R., Jiang, L., Zhang, X. and Yusuf, S. (2006) INTERHEART Study Investigators. Tobacco Use and Risk of Myocardial in Farction in 52 Countries in the INTERHEART Study: A Case-Control Study. Lancet, 368, 647-658. https://doi.org/10.1016/S0140-6736(06)69249-0

[2] Barnes, P.J. (2004) Mediators of Chronic Obstructive Pulmonary Disease. Pharmacological Reviews, 56, 515-548. https://doi.org/10.1124/pr.56.4.2

[3] Vurusaner, B., Poli, G. and Basaga, H. (2012) Tumor Suppressor Genes and ROS: Complex Networks of Interactions. Free Radical Biology \& Medicine, 52, 7-18. https://doi.org/10.1016/j.freeradbiomed.2011.09.035

[4] World Health Organization (2012) WHO Global Report: Mortality Attributable to Tobacco. http://www.who.int/tobacco/publications/surveillance/rep_mortality_attributable/e $\underline{\mathrm{n} /}$

[5] Kallio, T., Katariina, J.T., Eero, S.T., Maiju, H.T., Mauri, V.T., Iina, K.T., Tuuli, R.T., Tapani, V.T., Jorma, R.T.O. and Simell, T.O. (2010) Arterial Intima-Media Thickness, Endothelial Function, and Apolipoproteins in Adolescents Frequently Exposed to Tobacco Smoke. Circulation Cardiovascular Quality and Outcomes, 3, 196-203. https://doi.org/10.1161/CIRCOUTCOMES.109.857771

[6] Liu, P.Z., Kallio, H. and Yang, B.R. (2014) Flavonol Glycosides and Other Phenolic Compounds in Buds and Leaves of Different Varieties of Black Currant (Ribes nigrum L.) and Changes during Growing Season. Food Chemistry, 160, 180-189. https://doi.org/10.1016/j.foodchem.2014.03.056

[7] Aura, A.M., Martin-Lopez, P., O’Leary, K., Williamson, G., Oksman-Caldentey, K.M., Poutanen, K. and Santos-Buelga, C. (2005) In Vitro Metabolism of Anthocyanins by Human Gut Microflora. European Journal of Nutrition, 44, 133-142. https://doi.org/10.1007/s00394-004-0502-2

[8] McGhie, T.K. and Walton, M.C. (2007) The Bioavailability and Absorption of Anthocyanins: Towards a Better Understanding. Molecular Nutrition \& Food Research, 51, 702-713. https://doi.org/10.1002/mnfr.200700092

[9] Vepsäläinen, S., Koivisto, H., Pekkarinen, E., Mäkinen, P., Dobson, G., Mcdougall, G.J., Stewart, D., Haapasalo, A., Karjalainen, R.O., Tanila, H. and Hiltunen, M. (2013) Anthocyanin-Enriched Bilberry and Blackcurrant Extracts Modulate Amyloid Precursor Protein Processing and Alleviate Behavioral Abnormalities in the APP/PS1 Mouse Model of Alzheimer's Disease. Journal of Nutritional Biochemistry, 24, 360-370. https://doi.org/10.1016/j.jnutbio.2012.07.006

[10] Gopalan, A., Reuben, S.C., Ahmed, S., Darvesh, A.S., Hohmann, J. and Bishayee, A. (2012) The Health Benefits of Blackcurrants. Food \& Function, 3, 795-809. https://doi.org/10.1039/c2fo30058c

[11] Dohadwala, M.M., Holbrook, M., Hamburg, N.M., Shenouda, S.M., Chung, W.B., Titas, M., Kluge, M.A., Wang, N., Palmisano, J., Milbury, P.E., Blumberg, J.B. and Vim, J.A. (2011) Effects of Cranberry Juice Consumption on Vascular Function in Patients with Coronary Artery Disease. American Journal of Clinical Nutrition, 93, 934-940. https://doi.org/10.3945/ajcn.110.004242 
[12] Liu, R.H. (2004) Potential Synergy of Phytochemicals in Cancer Prevention: Mechanism of Action. Journal of Nutrition, 134, 3479S-3485S. https://doi.org/10.1093/jn/134.12.3479S

[13] Myung, S.K., Kim, Y, Ju, W., Choi, H.J. and Bae, W.K. (2010) Effects of Antioxidant Supplements on Cancer Prevention: Meta-Analysis of Randomized Controlled Trials. Annals of Oncology, 21, 166-179. https://doi.org/10.1093/annonc/mdp286

[14] Dohi, K., Satoh, K., Nakamachi, T., Yofu, S., Hiratsuka, K., Nakamura, S., Ohtaki, H., Yoshikawa, T., Shioda, S. and Aruga, T. (2007) Does Edaravone (MCI-186) Act as an Antioxidant and Neuroprotector in Experimental Traumatic Brain Injury? Antioxidants \& Redox Signaling, 8, 281-287. https://doi.org/10.1089/ars.2007.9.281

[15] Yanbaeva, D.G., Dentener, M.A., Creutzberg, E.C., Wesseling, G. and Wouters, E.F.M. (2007) Systemic Effects of Smoking. Chest, 131, 1557-1566.

https://doi.org/10.1378/chest.06-2179

[16] Muscat, J.E., Kleinman, W., Colosimo, S., Muir, A., Lazarus, P., Park, J. and Richie, J.P. (2004) Enhanced Protein Glutathiolation and Oxidative Stress in Cigarette Smokers. Free Radical Biology \& Medicine, 36, 464-470.

https://doi.org/10.1016/j.freeradbiomed.2003.11.018

[17] Gould, N.S., Min, E., Gauthier, S., Martin, R.J. and Day, B.J. (2011) Lung Glutathione Adaptive Responses to Cigarette Smoke Exposure. Respiratory Research, 12, 133-142. https://doi.org/10.1186/1465-9921-12-133

[18] Mccrea, K., Ensor, J., Nall, K., Bleecker, E. and Hasday, J. (1994) Altered Cytokine Regulation in the Lungs of Cigarette Smokers. American Journal of Respiratory and Critical Care Medicine, 150, 696-703. https://doi.org/10.1164/ajrccm.150.3.8087340

[19] Baharvand, M., Maghami, A.G., Azimi, S., Bastani, H., Ahmadieh, A. and Taghibakhsh, M. (2010) Comparison of Superoxide Dismutase Activity in Saliva of Smokers and Nonsmokers. The Southern Medical Journal, 103, 425-427. https://doi.org/10.1097/SMJ.0b013e3181d7e0d8

[20] Messner, B. and Bernhard, D. (2014) Smoking and Cardiovascular Disease: Mechanisms of Endothelial Dysfunction and Early Atherogenesis. Arteriosclerosis, Thrombosis, and Vascular Biology, 34, 509-515. https://doi.org/10.1161/ATVBAHA.113.300156

[21] Kanazawa, T., Osanai, T., Yin, X.Z., Yi, H.Z., Yi, H.Z., Onodera, K. and Metoki, H. (1996) Peroxidized Low-Density Lipoprotein with Four Kinds of Hydroperoxidized Cholesteryl Linoleate Estimated in Plasma of Young Heavy Smokers. Pathobiology, 64, 115-122. https://doi.org/10.1159/000164024

[22] Lykkesfeldt, J., Viscovich, M. and Poulsen, H.E. (2004) Plasma Malondialdehyde Is Induced by Smoking: A Study with Balanced Antioxidant Profiles. British Journal of Nutrition, 92, 203-206. https://doi.org/10.1079/BJN20041191

[23] Kashinakunti, S.V., Kollur, P., Kallaganada, G.S., Rangappa, M. and Ingin, J.B. (2011) Comparative Study of Serum MDA and Vitamin-C Levels in Non-Smokers, Chronic Smokers and Chronic Smokers with Acute Myocardial Infarction in Men. Journal of Research in Medical Sciences, 16, 993-998.

[24] Khaki-khatibi, F., Yaghoubi, A.R. and Rahbani, N.M. (2012) Study of Antioxidant Enzymes, Lipid Peroxidation, Lipid Profile and Immunologic Factor in Coronary Artery Disease in East Azarbijan. International Journal of Medicine and Biomedical Research, 1, 147-152. https://doi.org/10.14194/ijmbr.1210

[25] Ambrose, J.A. and Barua, R.S. (2004) The Pathophysiology of Cigarette Smoking and Cardiovascular Disease: An Update. Journal of the American College of Cardiology, 43, 1731-1737. https://doi.org/10.1016/j.jacc.2003.12.047 
[26] Agudo, A., Cabrera, L., Amiano, P., Ardanaz, E., Barricarte, A., Berenguer, T., Chirlaque, M.D., Dorronsoro, M., Jakszyn, P., Larrañaga, N., Martínez, C., Navarro, C., Quiros, J.R., Sanchez, M.J., Tormo, M.J. and Gonzalez, C.A. (2007) Fruit and Vegetable Intakes, Dietary Antioxidant Nutrients, and Total Mortality in Spanish Adults: Findings from the Spanish Cohort of the European Prospective Investigation into Cancer and Nutrition (EPIC-Spain). The American Journal of Clinical Nutrition, 85, 1634-1642. https://doi.org/10.1093/ajcn/85.6.1634

[27] Zhang, C.X., Ho, S.C., Chen, Y.M., Fu, J.H., Cheng, S.Z. and Lin, F.Y. (2009) Greater Vegetable and Fruit Intake Is Associated with a Lower Risk of Breast Cancer among Chinese Women. International Journal of Cancer, 125, 181-188.

https://doi.org/10.1002/ijc.24358

[28] Karlsen, A., Svendsen, M., Seljeflot, I., Sommernes, M.A., Sexton, J., Brevik, A., Erlund, I,, Serafini, M., Bastani, N., Remberg, S.F., Borge, G.I., Carlsen, M.H., Bøhn, S.K., Myhrstad, M.C., Dragsted, L.O., Duttaroy, A.K., Haffner, K., Laake, P., Drevon, C.A., Arnesen, H., Collins, A., Tonstad, S. and Blomhoff, R. (2011) Compliance, Tolerability and Safety of Two Antioxidant-Rich Diets: A Randomised Controlled Trial in Male Smokers. British Journal of Nutrition, 106, 557-571. https://doi.org/10.1017/S0007114511000353

[29] Lykkesfeldt, J., Christen, S., Wallock, L.M., Chang, H.H., Jacob, R.A. and Ames, B.N. (2000) Ascorbate Is Depleted by Smoking and Repleted by Moderate Supplementation: A Study in Male Smokers and Nonsmokers with Matched Dietary Antioxidant Intakes. The American Journal of Clinical Nutrition, 71, 530-536. https://doi.org/10.1093/ajen/71.2.530

[30] Kuiper, H.C., Langsdorf, B.L., Miranda, C.L., Joss, J., Jubert, C., Mata, J. and Stevens, J.F. (2010) Quantitation of Mercapturic Acid Conjugates of 4-hydroxy-2-nonenal and 4-oxo-2-nonenal Metabolites in a Smoking Cessation Study. Free Radical Biology \& Medicine, 48, 65-72.

https://doi.org/10.1016/j.freeradbiomed.2009.10.025

[31] Spitale, R.C., Cheng, M.Y., Chun, K.A., Gorell, E.S., Munoz, C.A., Kern, D.G., Wood, S.M., Knaggs, H.E., Wulff, J., Beebe, K.D. and Chang, A.L.S. (2012) Differential Effects of Dietary Supplements on Metabolomic Profile of Smokers versus Non-Smokers. Genome Medicine, 4, 14.

http://genomemedicine.com/content/4/2/14

[32] de Kok, T.M., de Waard, P., Wilms, L.C. and van Breda, S.G. (2010) Antioxidative and Antigenotoxic Properties of Vegetables and Dietary Phytochemicals: The Value of Genomics Biomarkers in Molecular Epidemiology. Molecular Nutrition \& Food Research, 54, 208-217. https://doi.org/10.1002/mnfr.200900288 